\title{
Hierarchy of Exchange Interactions in the Triangular-Lattice Spin Liquid $\mathrm{YbMgGaO}_{4}$
}

\author{
Xinshu Zhang, ${ }^{1}$ Fahad Mahmood, ${ }^{1, *}$ Marcus Daum, ${ }^{2}$ Zhiling Dun, ${ }^{3}$ Joseph A. M. Paddison, ${ }^{2,4}$ \\ Nicholas J. Laurita, ${ }^{1}$ Tao Hong, ${ }^{5}$ Haidong Zhou, ${ }^{3}$ N. P. Armitage, ${ }^{1}$ and Martin Mourigal ${ }^{2}$ \\ ${ }^{1}$ Institute for Quantum Matter, Department of Physics and Astronomy, \\ Johns Hopkins University, Baltimore, Maryland 21218, USA \\ ${ }^{2}$ School of Physics, Georgia Institute of Technology, Atlanta, Georgia 30332, USA \\ ${ }^{3}$ Department of Physics and Astronomy, University of Tennessee, Knoxville, Tennessee 37996, USA \\ ${ }^{4}$ Churchill College, University of Cambridge, Storey's Way, Cambridge CB3 ODS, United Kingdom \\ ${ }^{5}$ Neutron Scattering Division, Oak Ridge National Laboratory, Oak Ridge, Tennessee 37831, USA
}

(Received 24 August 2017; revised manuscript received 14 May 2018; published 2 July 2018; corrected 9 July 2018)

\begin{abstract}
The spin- $1 / 2$ triangular lattice antiferromagnet $\mathrm{YbMgGaO}_{4}$ has attracted attention recently as a quantum spin-liquid candidate with the possible presence of off-diagonal anisotropic exchange interactions induced by spin-orbit coupling. Whether a quantum spin liquid is stabilized or not depends on the interplay of various exchange interactions with chemical disorder that is inherent to the layered structure of the compound. We combine time-domain terahertz spectroscopy and inelastic neutron scattering measurements in the field-polarized state of $\mathrm{YbMgGaO}_{4}$ to obtain better insight of its exchange interactions. Terahertz spectroscopy in this fashion functions as a high-field electron spin resonance and probes the spin-wave excitations at the Brillouin zone center, ideally complementing neutron scattering. A global spin-wave fit to all our spectroscopic data at fields over $4 \mathrm{~T}$, informed by the analysis of the terahertz spectroscopy linewidths, yields constraints on the disorder-averaged $g$ factors and exchange interactions. Our results paint $\mathrm{YbMgGaO}_{4}$ as an easy-plane $X X Z$ antiferromagnet with the combined and necessary presence of subleading next-nearest neighbor and weak anisotropic off-diagonal nearest-neighbor interactions. Moreover, the obtained $g$ factors are substantially different from previous reports. This work establishes the hierarchy of exchange interactions in $\mathrm{YbMgGaO}_{4}$ from high-field data alone and thus strongly constrains possible mechanisms responsible for the observed spin-liquid phenomenology.
\end{abstract}

DOI: 10.1103/PhysRevX.8.031001

Subject Areas: Condensed Matter Physics

\section{INTRODUCTION}

Quantum spin liquids (QSLs) are exotic states of matter in which spins are highly correlated but remain dynamic down to zero temperature due to strong quantum fluctuations [1,2]. Many distinct QSL states have been proposed theoretically $[3,4]$ and classified according to their nonlocal (topological) properties [5]. Their detection, however, remains a central challenge for condensed matter physics [6], and relies on the presence of quantum entanglement in their ground state and fractional quasiparticles in their excitation spectra. While the former can be checked by numerics [7], the latter can be experimentally detected by thermodynamic techniques [8-10] or spectroscopic probes such as inelastic neutron scattering [11-17] and electronspin resonance [18-21].

\footnotetext{
*fahad@jhu.edu
}

Published by the American Physical Society under the terms of the Creative Commons Attribution 4.0 International license. Further distribution of this work must maintain attribution to the author(s) and the published article's title, journal citation, and DOI.
In spite of these recent breakthroughs, the most celebrated flavor of a QSL remains the resonating valencebond (RVB) state first proposed by Anderson in 1973 [22] for the spin-1/2 triangular-lattice Heisenberg antiferromagnet (TLHAF) and later extended to the square lattice in the context of cuprate superconductors [23]. However, precise numerical calculations for the spin-1/2 TLHAF, which take into account nearest-neighbor interactions only [24-26], indicate that quantum fluctuations are not enough to suppress magnetic ordering and the actual ground state is a noncollinear long-range ordered spin structure. Experiments on various spin- $S$ triangular-lattice antiferromagnets have overwhelmingly confirmed this picture [27-30], with a few noteworthy exceptions [31-33]. Several perturbations from the pure TLHAF have been proposed to enhance quantum fluctuations: next-nearest neighbor (NNN) interactions [34-38], ring-exchange terms [39,40], and anisotropic exchange [41-44], although it remains theoretically unclear if the latter mechanism alone can stabilize a QSL [45-48]. Nevertheless, anisotropic exchange interactions $[49,50]$ are known to generate new physics in rare-earth pyrochlores such as $\mathrm{Yb}_{2} \mathrm{Ti}_{2} \mathrm{O}_{7}$ [49,51-53], making it 
worthwhile to investigate their effect in other lattice geometries.

The newly discovered rare-earth triangular-lattice antiferromagnet $\mathrm{YbMgGaO}_{4}[54,55]$ appears to fulfill precisely this promise. The magnetic $\mathrm{Yb}^{3+}$ ions carry effective spin-1/2 moments in a symmetry environment allowing anisotropic exchange interactions [41,47] in the absence of antisymmetric (Dzyaloshinsky-Moriya) terms and magnetic defects, both of which are present in other two-dimensional QSL candidates such as herbertsmithite [56-58]. The immediate availability of single crystals [55] uncovered a QSL phenomenology in $\mathrm{YbMgGaO}_{4}$ characterized by the absence of spin ordering or freezing down to $T=100 \mathrm{mK}$ in muon spin relaxation measurements [59], much lower than the Curie-Weiss temperature $\theta_{W} \approx-4 \mathrm{~K}$, and a power-law behavior for the magnetic specific heat at low temperatures $[55,60]$. Perhaps the strongest evidence for a QSL in $\mathrm{YbMgGaO}_{4}$ came from inelastic neutron scattering measurements in zero field that unraveled a broad continuum of magnetic excitations across the entire Brillouin zone [61-63]. This continuum has been interpreted as being composed of fractional excitations from a U(1) QSL state with spinon Fermi surface $[42,44,62]$ or from a RVB-like state [63].

The absence of a magnetic contribution to the thermal conductivity [60], however, appears difficult to reconcile with the spinon Fermi surface interpretation. Additionally, the disordered occupancy of the intertriangular layers by $\mathrm{Mg}^{2+}$ and $\mathrm{Ga}^{3+}$ ions [54] appears to affect profoundly the $\mathrm{Yb}^{3+}$ ions with broadened crystal electric-field (CEF) levels [61,64], a distribution of $g$ tensors [64], and a broadened magnetic excitation spectrum at high fields [61]. The impact of disorder on the $\mathrm{YbMgGaO}_{4}$ exchange Hamiltonian and, therefore, whether the ground state is a QSL or not, remains an outstanding issue $[47,48]$. In fact, the nature of the dominant exchange interactions in $\mathrm{YbMgGaO}_{4}$ is also controversial. While the overall planar anisotropy is clear from susceptibility measurements $[55,62]$, both antiferromagnetic next-nearest-neighbor terms $\left(J_{2}\right)$ [61] and nearest-neighbor anisotropic offdiagonal exchanges (so-called $J_{1}^{ \pm \pm}$and $J_{1}^{z \pm}$ ) $[43,65]$ have been proposed as extensions from the $X X Z$ model. Comprehensively determining the exchange interactions in $\mathrm{YbMgGaO}_{4}$ is of fundamental importance in deciphering the nature of its ground state.

Here, we combine time-domain $\mathrm{THz}$ spectroscopy (TDTS) with inelastic neutron scattering (INS) measurements in the field-polarized state of $\mathrm{YbMgGaO}_{4}$ to extract values of the disorder-averaged exchange interactions from a global fit to spin-wave spectra measured for different field directions and scattering wave vectors including the Brillouin-zone center ( $\Gamma$ point). We note that the randomness of the interlayer $\mathrm{Mg} / \mathrm{Ga}$ atoms in $\mathrm{YbMgGaO}_{4}$ causes displacements of the $\mathrm{Yb}$ atoms [55] leading to modulations (disorder) in the $g$ tensors [64] and exchange interactions
[66]. Thus, the values of the exchange parameters we determine in this work are averaged over these intrinsic disorder effects. Previous high-field INS measurements were limited to wave vectors around the antiferromagnetic zone boundary [61] while previous $X$-band electron spin resonance (ESR) measurements [55] were intrinsically limited to small fields $(\lesssim 0.4 \mathrm{~T})$ below the field-polarized regime. In contrast, high-resolution TDTS (see, e.g., Ref. [67]) functioning as high-field ESR on magnetic insulators allows an accurate determination of magnetic excitations in fields comparable to the saturation field in $\mathrm{YbMgGaO}_{4}[55,61]$. Since the wavelength of $\mathrm{THz}$ radiation is much greater than lattice constants, TDTS measures excitations in the first Brillouin zone with zero-momentum transfer (i.e., the $\mathbf{q}=0$ response), which is impossible with neutron scattering. Inclusion of the high-field TDTS data allows a substantial refinement of the Hamiltonian parameters compared to Ref. [61]. Moreover, an analysis of TDTS linewidths further constrains the anisotropic exchange interactions. In the context of prevailing models our results suggest that both NNN and off-diagonal anisotropic exchange interactions are present in $\mathrm{YbMgGaO}_{4}$ - with pseudodipolar terms subleading compared with the $X X Z$ part of the model — and that this nominal set of exchanges may lie closer to a spin-liquid regime than previously thought $[47,61]$. When combined with the likely presence of exchange disorder, the extent of which remains to be determined, our work strongly constrains the nature of the $\mathrm{YbMgGaO}_{4}$ ground state and points towards a more intricate underlying scenario than originally reported [62].

\section{METHODS}

\section{A. Experimental methods}

The $\mathrm{YbMgGaO}_{4}$ crystal (space group $R \overline{3} \mathrm{~m}$ ) used in this work was grown by the floating-zone technique [see Supplemental Material (SM) Sec. I for details [68]] as reported in Ref. [61] and cut with a diamond blade to present a $c$-axis facet, where the $c$ axis is orthogonal to the triangular $a b$ plane of $\mathrm{Yb}^{3+}$ ions. TDTS measurements were performed in a custom-built polarization modulation setup with a frequency range of $0.2-2 \mathrm{THz}(0.83-8.3 \mathrm{meV})$ (see SM Sec. II [68]). The complex THz transmission of a $4 \times 4 \times 1 \mathrm{~mm}^{3}$ single crystal was measured down to $1.6 \mathrm{~K}$ (see SM Sec. VII [68]) with external fields up to $\mathbf{H}=6.8 \mathrm{~T}$ in both the Faraday $(\mathbf{k} \| \mathbf{H})$ and Voigt $(\mathbf{k} \perp \mathbf{H})$ geometries, where $\mathbf{k}$ is the direction of light propagation. In both cases, the $\mathrm{THz}$ pulse ac magnetic field $\mathbf{h}$ was applied along $\mathbf{a}^{*}$ with $\mathbf{h} \perp \mathbf{H}$, and thus the $\mathbf{H} \| \mathbf{c}$ and $\mathbf{H} \| \mathbf{a}$ orientations were probed in Faraday and Voigt geometries, respectively.

INS experiments were performed on the cold neutron chopper spectrometer (CNCS) [69] at the Spallation Neutron Source (SNS), and on the cold triple-axis spectrometer CTAX at the High Flux Isotope Reactor (HFIR), both at Oak Ridge National Laboratory. Experiments were 

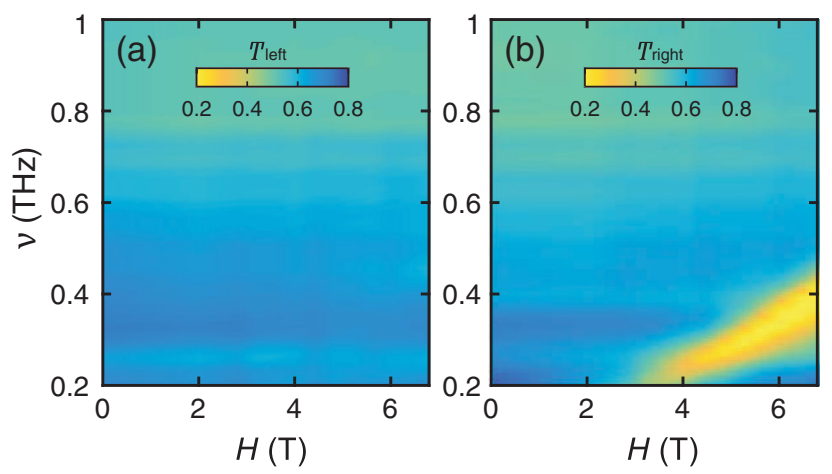

FIG. 1. Transmission amplitude for (a) left- and (b) rightcircularly polarized $\mathrm{THz}$ light as a function of frequency $\nu$ and field $H_{\mathrm{dc}}$ in the Faraday geometry; i.e., $H_{\mathrm{dc}} \| c$ at $T=5 \mathrm{~K}$. The transmission curves were measured at 0,1 , and $2 \mathrm{~T}$, and then at every $0.4 \mathrm{~T}$ from 2.8 to $6.8 \mathrm{~T}$. The bright yellow feature in (b) indicates the absorption due to the $\mathbf{q}=0$ excitation.

performed on the same crystals as Ref. [61] with a magnetic field of $H=7.8 \mathrm{~T}$ along the crystal $c$ axis and $T \approx 0.06 \mathrm{~K}$ on CNCS, and at $H=10.8 \mathrm{~T}$ along the crystal $a$ axis and $T \approx 0.32 \mathrm{~K}$ on CTAX. The INS data taken at CNCS are the same as published in Ref. [61], but now analyzed together with the TDTS data. Given the broad spectra, even at high fields, the energy of magnetic excitations for a given wave vector was determined by fitting the maximum in scattering intensity (see Ref. [61] and SM Sec. III [68]).

\section{B. Analysis of the TDTS data}

In the field-polarized state in the Faraday geometry, the linearly polarized $\mathrm{THz}$ pulse becomes elliptically polarized as it passes through the sample due to spins precessing around the applied field direction. The complex transmission is represented by a $2 \times 2$ Jones matrix. Because of the threefold rotational symmetry of the lattice, this reduces to an antisymmetric matrix for the transmission of a linearly polarized pulse [70]. By diagonalizing this antisymmetric matrix, we can convert the transmission matrix from the linear basis into a circular basis (see SM Sec. II [68]), which naturally corresponds to eigenstates of the transmission in the Faraday geometry [51]. In this manner we can separate the transmission of a left (LCP) and right (RCP) circularly polarized $\mathrm{THz}$ pulse.

Figures 1(a) and 1(b) show such transmission magnitudes for LCP and RCP THz light, respectively, and as a function of frequency and magnetic field at a temperature of $T=5 \mathrm{~K}$. The bright yellow feature in Fig. 1(b) shows that only one particular helicity of the light (RCP) is strongly absorbed. This indicates the presence of welldefined spin-wave excitations with energy linearly dependent on the applied field. In a field-polarized regime in the Faraday geometry, the direction of spin precession is determined by the orientation of the applied $H_{\mathrm{dc}}$ field and it is therefore natural to expect an absorption for only one helicity and not the other. Indeed, our experiments confirm that when the polarity of the $H_{\mathrm{dc}}$ field is flipped, only LCP light is absorbed (see SM Sec. IV [68]). This confirms that the absorption line observed in Fig. 1(b) originates from spin-wave excitations in the field-polarized regime of $\mathrm{YbMgGaO}_{4}$. The observed absorption is the only feature in the data that is affected by the $H_{\mathrm{dc}}$ field and temperature, suggesting the featureless background has a nonmagnetic origin and is likely the low-energy tail of a crystal field level absorption (see SM Sec. V [68]).

The featureless nature of the background allows us to extract the frequency-dependent magnetic susceptibility $\tilde{\chi}(\nu)$ as follows. The complex transmission of a particular eigenpolarization (LCP or RCP light) is given by the relation $\tilde{T}(\nu)=\left[4 \tilde{n} /(\tilde{n}+1)^{2}\right] \exp [i 2 \pi \nu d(\tilde{n}-1) / c]$, where $\tilde{n}=\sqrt{\tilde{\epsilon} \tilde{\mu}}$ is the complex index of refraction, $\tilde{\epsilon}$ is the dielectric constant, $\tilde{\mu}=1+\tilde{\chi}$, and $d$ is the sample thickness. We determine $\tilde{n}$ using the Newton-Raphson method and then isolate $\tilde{\chi}$ by measuring the sample at a reference temperature at which the spectrum does not show any signatures of the spin-wave absorption [52]. At this
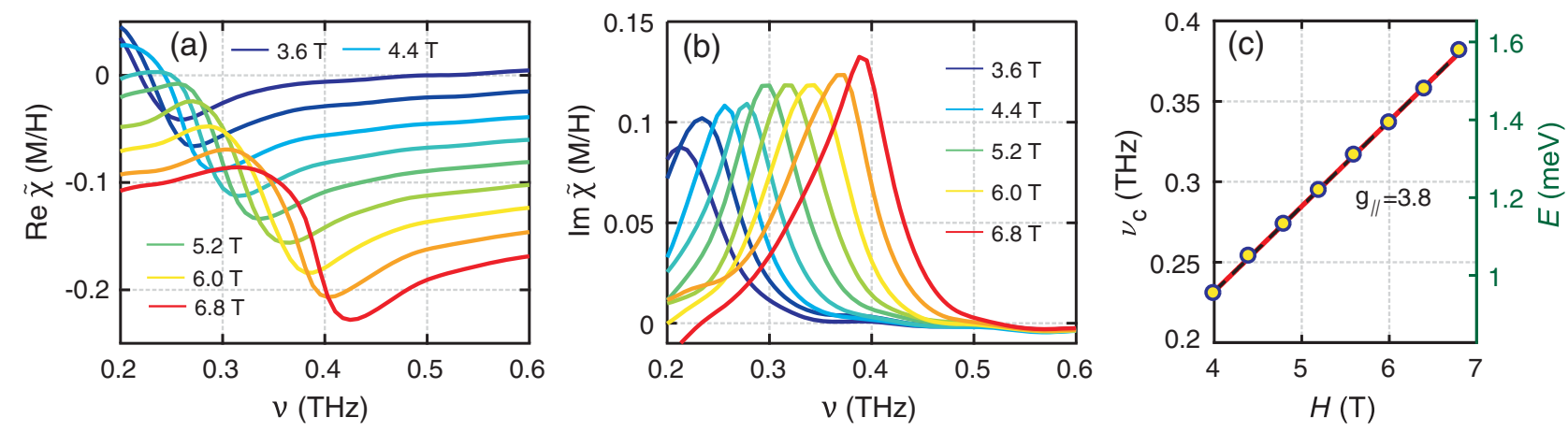

FIG. 2. Real (a) and imaginary (b) parts of the magnetic susceptibility $\tilde{\chi}(\nu)$ for right-circularly polarized light at different fields $H_{\mathrm{dc}} \| c$ at $5 \mathrm{~K} . \tilde{\chi}(\nu)$ is obtained by referencing the TDTS data to the spectra at $100 \mathrm{~K}$. Spectra in (a) are offset vertically by 0.02 for clarity. (c) Resonant frequency $\left(\nu_{c}\right)$ at $5 \mathrm{~K}$ versus magnetic field for magnetic excitation peaks in (b) in the Faraday geometry $H_{\mathrm{dc}} \| c$. Yellow circles represent the data. Red line is a linear best fit to extract $g_{\|}$. The black dashed line represents the global fit to the data (see Fig. 3 ). Error bars in (c) are smaller than the marker size. 

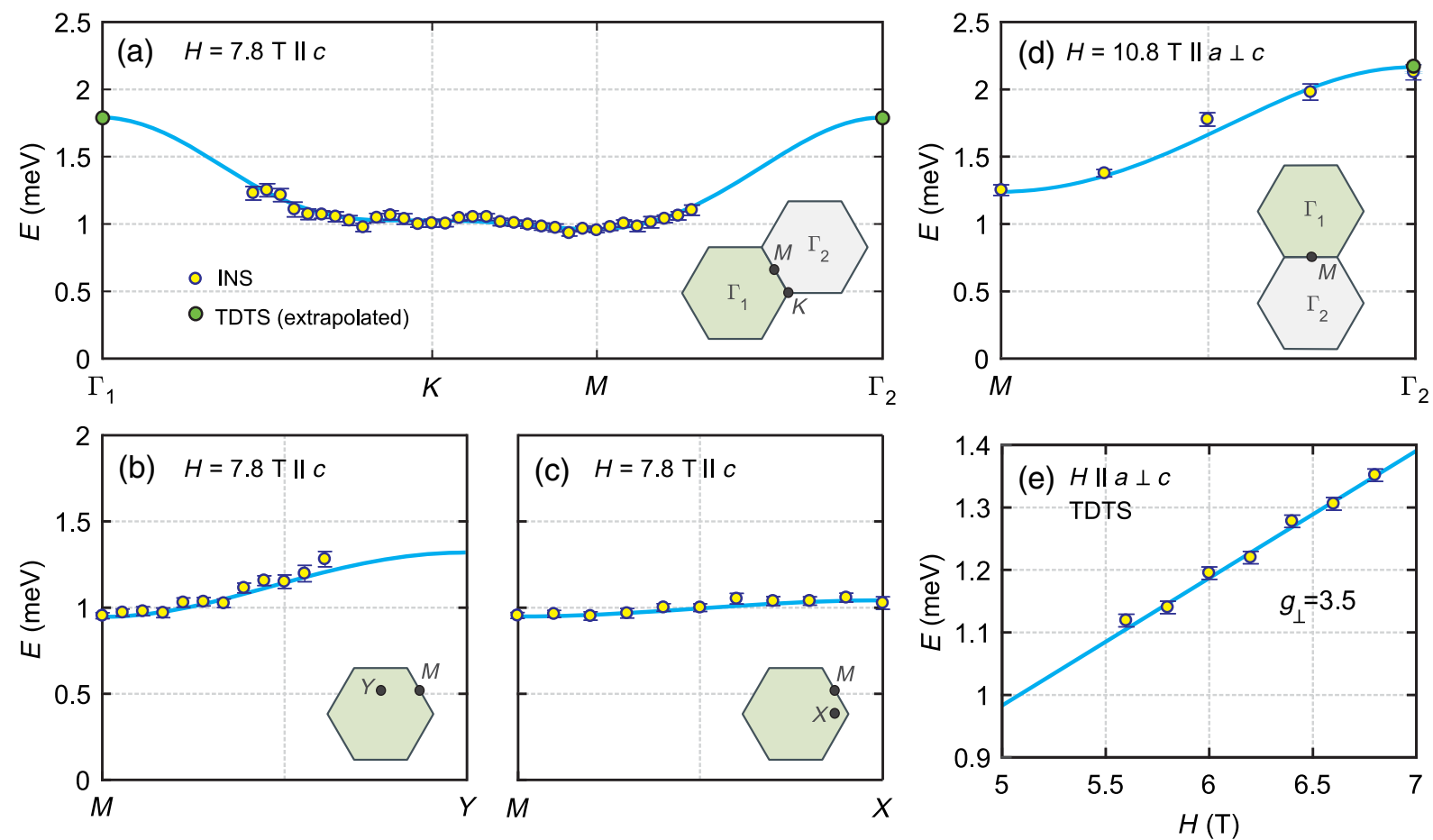

FIG. 3. (a)-(c) Energy dependence of spin-wave excitations in the field-saturated state from INS data along high-symmetry directions of the triangular Brillouin zone. The sample was cooled by a dilution refrigerator of base temperature $T=0.06 \mathrm{~K}$ in a magnetic field $H_{\mathrm{dc}} \| c=7.8 \mathrm{~T}$. Solid blue lines show the results of the global fit using model C (see Table I). Green circles at the $\Gamma$ points are the TDTS data from Fig. 2(c) extrapolated to $7.8 \mathrm{~T}$ and included for display purposes. Error bars for these extrapolated data are smaller than the marker size. (d) INS data measured at $T=0.32 \mathrm{~K}$ in a field $H_{\mathrm{dc}} \| a=10.8 \mathrm{~T}$. Yellow circles show the position of the maximum intensity at each wave vector as determined from fits to the energy-dependent data. Green circle at the $\Gamma_{2}$ point shows the TDTS data extrapolated to $10.8 \mathrm{~T}$. (e) Energy versus field for spin-wave excitations at $T=5 \mathrm{~K}$ in the TDTS Voigt geometry, i.e., $H_{\mathrm{dc}} \| a$. Yellow circles represent the resonant energy of the spin-wave peaks in the TDTS spectra (see SM [68]). Solid blue lines in (d) and (e) show the results of the global fit using model C (Table I). Note that the INS data in (a)-(c) are the same as published in Ref. [61].

reference temperature (100 K in this case; see SM Sec. VI [68]), $\tilde{\chi}$ can be taken to be zero and so $\tilde{n}_{100 \mathrm{~K}}=\sqrt{\tilde{\epsilon}}$. Thus, the low-temperature magnetic susceptibility is given by $\tilde{\chi}=\left(\tilde{n}_{5 \mathrm{~K}} / \tilde{n}_{100 \mathrm{~K}}\right)^{2}-1[71]$.

Figures 2(a) and 2(b) show the real and imaginary parts of $\tilde{\chi}(\nu)$, respectively, at different fields and $T=5 \mathrm{~K}$ in the Faraday geometry. Peaks in $\operatorname{Im} \tilde{\chi}(\nu)$ correspond to the spinwave excitations in the $\mathbf{q}=0$ limit. By fitting the data at each field with a Lorentzian (see SM Sec. VII [68]) we extract the resonant energy $(E)$ of the spin-wave absorption. The resulting $E$ versus $H_{\mathrm{dc}}$ plot is shown in Fig. 2(c). The peak widths and resonant frequencies show little temperature dependence between 1.6 and $40 \mathrm{~K}$ (see SM Sec. VII [68]). Similar analysis (see SM Sec. VIII) is done for TDTS in the Voigt geometry to extract the spin-wave energies for field $H_{\mathrm{dc}}$ along the $a$ axis (reported in Fig. 3). These TDTS data are then combined with INS data to extract the disorder-averaged exchange interactions in a global fit, as discussed below.

\section{High-field spin-wave theory analysis}

An effective spin-1/2 Hamiltonian relevant for $\mathrm{YbMgGaO}_{4}$ has been given by $[41,42,55,61]$

$$
\begin{aligned}
\mathcal{H}= & \sum_{\langle i, j\rangle}\left[J_{1}^{z z} S_{i}^{z} S_{j}^{z}+J_{1}^{ \pm}\left(S_{i}^{+} S_{j}^{-}+S_{i}^{-} S_{j}^{+}\right)\right. \\
& +J_{1}^{ \pm \pm}\left(\gamma_{i j} S_{i}^{+} S_{j}^{+}+\gamma_{i j}^{*} S_{i}^{-} S_{j}^{-}\right) \\
& \left.-\frac{i J_{1}^{z \pm}}{2}\left(\gamma_{i j}^{*} S_{i}^{+} S_{j}^{z}-\gamma_{i j} S_{i}^{-} S_{j}^{z}+\langle i \leftrightarrow j\rangle\right)\right] \\
& +\sum_{\langle\langle i, j\rangle\rangle}\left[J_{2}^{z z} S_{i}^{z} S_{j}^{z}+J_{2}^{ \pm}\left(S_{i}^{+} S_{j}^{-}+S_{i}^{-} S_{j}^{+}\right)\right] \\
& -\mu_{0} \mu_{B} \sum\left[g_{\perp}\left(H^{x} S_{i}^{x}+H^{y} S_{i}^{y}\right)+g_{\|} H^{z} S_{i}^{z}\right],
\end{aligned}
$$

where $S^{ \pm}=S^{x} \pm i S^{y}, g_{\|}$and $g_{\perp}$ are components of the $g$ tensor parallel and perpendicular to the $c$ axis, complex numbers $\gamma_{i j}$ are defined in Ref. [55], and brackets \langle\rangle and $\langle\langle\rangle\rangle$ represent nearest and next-nearest neighbor pairs, respectively. The exchange interactions $J^{z z}$ and $J^{ \pm}$originate from the standard $X X Z$ model, with subscripts 1 and 2 indicating the nearest and next-nearest neighbor interactions, respectively. We also include symmetry-allowed bond-dependent interactions $J^{ \pm \pm}$and $J^{z \pm}$ (also known as pseudodipolar interactions), which have a spin-orbit origin. The pseudodipolar interactions between next-nearest 
neighbors are neglected since they are supposed to be small [72,73]. For large applied fields and assuming a fieldpolarized state, the above Hamiltonian can be solved to yield the spin wave dispersions for external fields along the $c$ and $a$ axes (see SM [68]).

By setting $\mathbf{q}=0$, the spin-wave excitations for the Faraday and Voigt geometry can be simplified to

$$
\begin{aligned}
E\left(H^{z}\right) & =\mu_{0} \mu_{B} g_{\|} H^{z}-3\left(J_{1}^{z z}+J_{2}^{z z}\right)+6\left(J_{1}^{ \pm}+J_{2}^{ \pm}\right), \\
E\left(H^{x}\right)= & \left\{\left[\mu_{0} \mu_{B} g_{\perp} H^{x}+\frac{3}{2}\left(J_{1}^{z z}+J_{2}^{z z}\right)-3\left(J_{1}^{ \pm}+J_{2}^{ \pm}\right)\right]^{2}\right. \\
& \left.-\left|\frac{3}{2}\left(J_{1}^{z z}+J_{2}^{z z}\right)-3\left(J_{1}^{ \pm}+J_{2}^{ \pm}\right)\right|^{2}\right\}^{1 / 2} .
\end{aligned}
$$

According to Eq. (2), the field dependence in the Faraday geometry is particularly simple and depends only on $g_{\|}$. From a simple linear fit to the data in Fig. 2(c) we obtain $g_{\|}=3.81(4)$. Note that the effective $g$ tensor here is a property of a single $\mathrm{Yb}^{3+}$ ion and as such is independent of the exchange interactions [49]. This allows us to treat $g_{\|}$as a fixed parameter in the subsequent global fit. In contrast, $E\left(H^{x}\right)$ is not linearly related to $g_{\perp}$ [Eq. (3)] and so $g_{\perp}$ must be extracted simultaneously with the exchange constants of Eq. (1). One expects the present measurements of the $g$ factor to be considerably more accurate than previous $X$-band ESR results [55] due to greater than a tenfold increase in the magnitude of the magnetic fields. The expressions for the dispersions with $\mathbf{q} \neq 0$, as relevant for neutron-scattering measurements, are given in Refs. [43,61] and SM Sec. III [68].

\section{RESULTS}

\section{A. Fits to spin-wave energies}

We now turn to our central result, which is to refine the parameters of $\mathrm{YbMgGaO}_{4}$ by combining all the data at hand. In Fig. 3, we show the spin-wave dispersion obtained through INS [Figs. 3(a)-3(d)] as well as the TDTS data in Voigt geometry [Fig. 3(e)]. The entire data set is fit simultaneously to the spin-wave dispersions in the fieldsaturated state obtained from the Hamiltonian in Eq. (1). There are six target parameters for this global fit, which are $J_{1}^{z z}, J_{1}^{ \pm}, J_{1}^{ \pm \pm},\left|J_{1}^{z \pm}\right|$, the ratio $J_{2} / J_{1}$, and $g_{\perp}$ with a fixed $g_{\|}=3.81$. Note that only the magnitude of $J_{1}^{z \pm}$ can be determined from the spin-wave dispersions (see SM Sec. IX [68]). The signs of the other $J$ terms obtained from the fit are verified by starting from both negative and positive initial values. As spin-space anisotropy is primarily a property of the effective spin- $1 / 2$ doublet of $\mathrm{Yb}^{3+}$, we adopt the same overall $X X Z$ exchange anisotropy for both nearest neighbor and next-nearest neighbor interactions; i.e., $J_{2}^{z z} / J_{2}^{ \pm}=J_{1}^{z z} / J_{1}^{ \pm}$. This reasonable assumption helps in reducing the size of the parameter space for the global fit.

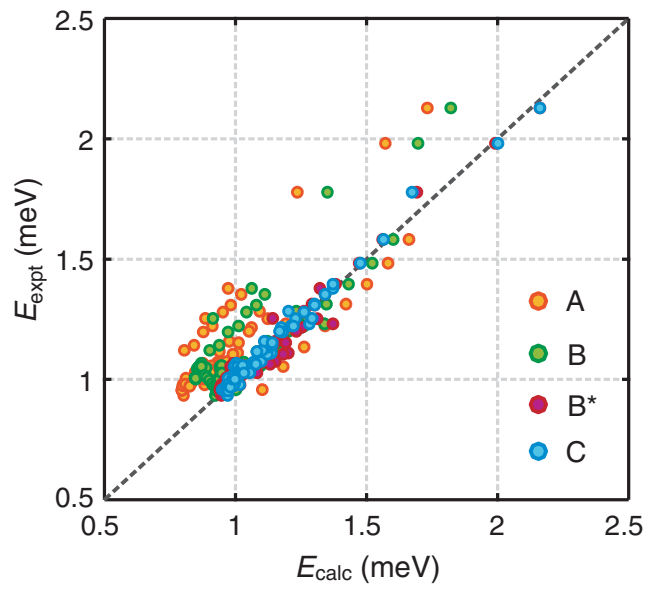

FIG. 4. Experimental versus calculated spin-wave energies, where the dashed line denotes $E_{\text {expt }}=E_{\text {calc }}$. Fitted exchange parameters for models A, B, and C are listed in Table I. Models A (Ref. [61]), B (Ref. [43]), and C (global fit to the TDTS and THz data) are represented by orange, green, and blue symbols, respectively. Model $\mathrm{B}^{*}$ (global fit to data under the $J_{2}=0$ constraint) is represented by pink symbols.

We obtain an excellent fit to the data using the above model [see lines in Fig. 3 and spread in Fig. 4] and report our global fitting parameters in Table I (model C). As discussed above, the extracted parameters in this work are disorder averaged. The $\chi^{2}$ goodness of fit and correlation plots of these parameters are shown in the SM Sec. X [68]. We note that the above fit is performed with the constraint $\left|J_{1}^{z z}\right|>\left|J_{1}^{z \pm}\right|$. Without this, we obtain $\left|J_{1}^{z \pm}\right|=0.45$, which we regard as unphysical as it is nearly 3 times larger than $J_{1}^{z z}$ and also considerably outside the bounds set by the linewidth analysis performed below.

In previous works, two different sets of parameters have been proposed for the exchange interactions of $\mathrm{YbMgGaO}_{4}$. The first set [61], model A in Table I, includes both nearest and next-nearest neighbor interactions but sets

TABLE I. Exchange parameters for different models derived from fitting the spin-wave dispersions. Models A and B are from Ref. [61] and Ref. [43], respectively. Model C is from our global fit to the TDTS and INS data. Model B* is from a global fit to the data by ignoring NNN interactions, i.e., $J_{2}=0$. Uncertainties in the values represent the $99.7 \%$ confidence interval (3 s.d.) in extracting the fitting parameters.

\begin{tabular}{lllll}
\hline \hline Model & \multicolumn{1}{c}{$\mathrm{A}$} & \multicolumn{1}{c}{$\mathrm{B}$} & \multicolumn{1}{c}{$\mathrm{B}^{*}$} & \multicolumn{1}{c}{$\mathrm{C}$} \\
\hline$J_{1}^{z z}(\mathrm{meV})$ & 0.126 & 0.164 & $0.151(5)$ & $0.149(5)$ \\
$J_{1}^{ \pm}(\mathrm{meV})$ & 0.109 & 0.108 & $0.088(3)$ & $0.085(3)$ \\
$J_{1}^{ \pm \pm}(\mathrm{meV})$ & 0.013 & 0.056 & $0.13(2)$ & $0.07(6)$ \\
$\left|J_{1}^{z \pm}\right|(\mathrm{meV})$ & 0 & 0.098 & $0.1(1)$ & $0.1(1)$ \\
$J_{2} / J_{1}$ & 0.22 & 0 & 0 & $0.18(7)$ \\
$g_{\|}$ & 3.72 & 3.72 & $3.81(4)$ & $3.81(4)$ \\
$g_{\perp}$ & 3.06 & 3.06 & $3.53(5)$ & $3.53(5)$ \\
\hline \hline
\end{tabular}


$J_{1}^{z \pm}=0$ based on previous $X$-band ESR results [55]. The second set [43], model B in Table I, includes only nearestneighbor interactions, with the $J_{2}=0$ constraint argued as a consequence of the localized nature of $\mathrm{Yb}^{3+} 4 f$ electrons $[43,65]$. Both models used the $g$ factors determined from low-frequency $X$-band ESR. Model $\mathrm{C}$ is the new best fit from our spin-wave analysis. To compare these models, we plot in Fig. 4 the experimental versus calculated spin-wave energies for each of models A, B, and C. Clearly, the points obtained for our model $\mathrm{C}$ lie significantly closer to the $E_{\text {expt }}=E_{\text {calc }}$ line than models $\mathrm{A}$ and $\mathrm{B}$, highlighting the advantage of our enriched data sets over previous works.

Our global analysis yields finite NNN interactions with $J_{2} / J_{1}=0.18(7)$ and a slightly subdominant pseudodipolar $J_{1}^{ \pm \pm} \approx 0.07 \mathrm{meV}$ interaction. The large fitting error bars on some parameters of the model reveal the high degree of correlation between $J_{2}$ and $J_{1}^{ \pm \pm}$and the very weak sensitivity of our fit to $\left|J_{1}^{z \pm}\right|$. This is apparent in the $\chi^{2}$ goodness of fit 2D plots (see SM Sec. X [68]) in the parameter spaces of $J_{1}^{ \pm \pm}$versus $\left|J_{1}^{z \pm}\right|$ and $J_{1}^{ \pm \pm}$versus $J_{2} / J_{1}$, respectively. The poor sensitivity to $\left|J_{1}^{z \pm}\right|$ is also clear from the analytical expression for the field-polarized spin-wave dispersion with field along the $a$ axis (see SM Sec. IX [68]).

Given the correlation between $J_{1}^{ \pm \pm}$and $J_{2}$, it is natural to analyze how our results change by enforcing the constraint $J_{2}=0$ while leaving all other parameters free. This leads to model $\mathrm{B}^{*}$, which resembles model $\mathrm{C}$ except for a larger $J_{1}^{ \pm \pm}=0.13(2) \mathrm{meV}$, which is now comparable to the dominant $J_{1}^{z z}$ exchange (see Table I). This model gives only a slightly worse fit to the spin-wave data than model C, as can been seen from a plot of the experimental against calculated spin-wave energies in Fig. 4. While a unique set of exchange parameters cannot be obtained from the above analysis, a definitive hierarchy of interactions nevertheless emerges. It yields easy-plane $X X Z$ terms for $\mathrm{YbMgGaO}_{4}$ with $J_{1}^{z z}=0.15(1) \mathrm{meV}, \Delta=J_{1}^{z z} / 2 J_{1}^{ \pm}=0.9(1)$, a subleading pseudodipolar term $J_{1}^{ \pm \pm} / J_{1}^{z z} \leq 1$, and a small NNN exchange $J_{2}^{z z}=0.03(1) \mathrm{meV}$.

\section{B. Further constraints: TDTS line shapes}

To further constrain these parameters, we require additional information. For that, we analyzed the linewidths of the spin-wave peaks in the TDTS data of Fig. 2. As noted above, TDTS functions here as high-field ESR [57]. ESR linewidths are strongly sensitive to the magnitude of the off-diagonal anisotropic interactions $J_{1}^{ \pm \pm}$and $J_{1}^{z \pm}$ of our model. The analysis proceeds using the formalism of KuboTomita $[55,74,75]$, which relates the width of the ESR absorption line to commutators that depend only on the anisotropic exchange interactions. This perturbative result is valid for small anisotropic exchanges when temperature and magnetic field are larger than the dominant exchange, conditions that are realized in our high-field TDTS

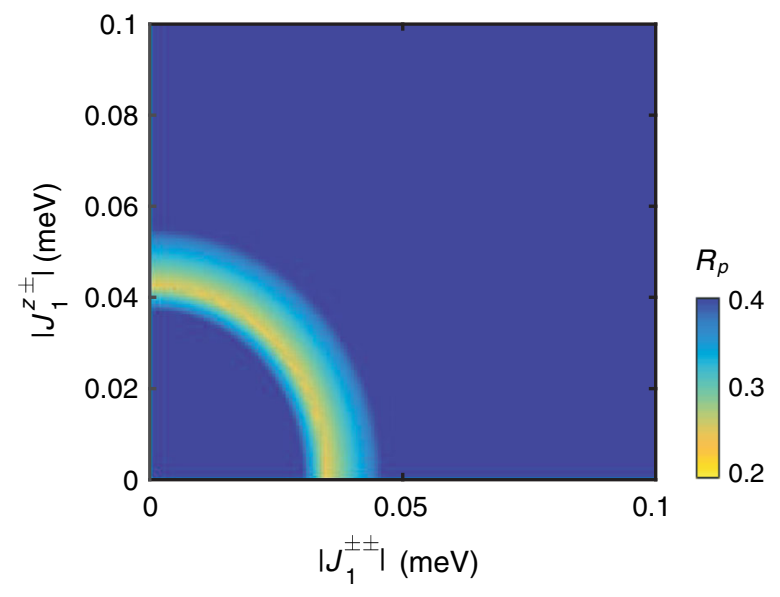

FIG. 5. Deviation $R_{p}$ of the experimental TDTS spin-wave resonance linewidths from the theoretically calculated ones as a function of the pseudodipolar interactions $\left|J_{1}^{z \pm}\right|$ and $\left|J_{1}^{ \pm \pm}\right|$. This sets an upper bound on the pseudodipolar interactions in a model with $J_{2}=0$.

experiments. The analysis [55] shows the Lorentzian width $\Delta_{\text {calc }}=\sqrt{2 \pi M_{2}^{3} / M_{4}}$, where $M_{2}$ and $M_{4}$ are the second and fourth moment of the anisotropic part of the Hamiltonian (see SM Sec. 11 [68]).

Here, we use this formalism to show that the observed spin-wave linewidths cannot be reconciled with the large anisotropic exchange terms suggested by model $\mathrm{B}^{*}$ and therefore $J_{2}$ must be nonzero. In Fig. 5, we plot the deviation between the calculated and the experimental spin-wave resonance linewidths in both the Faraday $\Delta_{f}$ and Voigt $\Delta_{v}$ geometry as a function of $\left|J_{1}^{z \pm}\right|$ and $\left|J_{1}^{ \pm \pm}\right|$. We analyze the case of $J_{2}=0$ that is relevant for model B*. We define a function $R_{p}=\frac{1}{2}\left[\left|\left(\Delta_{f}-\Delta_{\text {calc }}\right) / \Delta_{f}\right|+\right.$ $\left.\left|\left(\Delta_{v}-\Delta_{\text {calc }}\right) / \Delta_{v}\right|\right]$, that is minimal along the yellow contour in Fig. 5, for which a rough analytical description is $\sqrt{\left|J_{1}^{z \pm}\right|^{2}+\left|J_{1}^{ \pm \pm}\right|^{2}} \approx 0.04(1) \mathrm{meV}$. This calculation assumes that all the broadening of the $\mathrm{THz}$ data comes from exchange anisotropy with no distribution of $g$ factors beyond the values determined above. In fact, a relatively large spread in the latter values, $\Delta g_{\|} / \bar{g}_{\|} \approx 0.3$ and $\Delta g_{\perp} / \overline{g_{\perp}} \approx 0.1$, has been demonstrated by a careful analysis of CEF excitations linewidths [64] and must also play a role in the line shapes observed here. Our analysis of the highfield TDTS line shapes therefore provides an upper bound on any off-diagonal anisotropic exchanges $\left|J_{1}^{z \pm}\right|$ and $\left|J_{1}^{ \pm \pm}\right|$. The parameters determined in model $\mathrm{B}^{*}$ are incompatible with this bound, and so model $\mathrm{B}^{*}$ is ruled out as a realistic description of the exchange parameters of $\mathrm{YbMgGaO}_{4}$.

Taken together, our results strongly favor an easy-plane $X X Z$ scenario for $\mathrm{YbMgGaO}_{4}$ with the combination of finite $J_{2}$ and relatively small pseudodipolar exchanges. Thus, the refinement of model $\mathrm{C}$ with a range of best 
possible disorder-averaged parameter values we found for $\mathrm{YbMgGaO}_{4}$ can be summarized as a model $\mathrm{C}^{*}$ :

$$
\begin{aligned}
J_{1}^{z z} & =0.149(5) \mathrm{meV}, \quad J_{1}^{ \pm}=0.085(3) \mathrm{meV}, \\
J_{2} / J_{1} & =0.18(7), \quad \sqrt{\left|J_{1}^{ \pm \pm}\right|^{2}+\left|J_{1}^{z \pm}\right|^{2}} \lesssim 0.05 \mathrm{meV}, \\
g_{\|} & =3.81(4), \quad g_{\perp}=3.53(5) .
\end{aligned}
$$

The above analysis assumes that finite $J_{2}$ will also give an additional contribution to the linewidth, and hence the bounds on $\left|J_{1}^{ \pm \pm}\right|$and $\left|J_{1}^{z \pm}\right|$ for $J_{2}=0$ represent the maximum values that these parameters can take. We note that the bound determined for $\left|J_{1}^{z \pm}\right|$ justifies the earlier constraint of $\left|J_{1}^{z z}\right|>\left|J_{1}^{z \pm}\right|$ in determining the parameters for model $\mathrm{C}$ in Table I.

\section{Discussion}

Experiments using other techniques, such as unpolarized [61] and polarized [65] neutron diffraction in zero field and low temperatures, may help to constrain the values further. Indeed, classical Monte Carlo simulations for the instantaneous spin structure factor $S(\mathbf{Q})$ reveal that either large $J_{1}^{ \pm \pm}$ or relatively small $J_{2} \sim 0.2 J_{1}$ yields correlations peaked at the $M$ point of the triangular-lattice Brillouin zone [61]. Maximal correlations at the $M$ point have been reported in all neutron scattering investigations to date $[61-63,65]$. In this regard, we note that Monte Carlo simulations with a large $J_{1}^{ \pm \pm}$yield a strong modulation in $S(\mathbf{Q})$ from the first to the second Brillouin zone [61]. To the best of our knowledge, such a modulation has not yet been observed experimentally, which appears consistent with the relatively small $J_{1}^{ \pm \pm}$term indicated by our spin-wave resonance linewidth analysis. In general, the sensitivity of $S(\mathbf{Q})$ to spin anisotropy arises because neutrons scatter only from spin components perpendicular to $\mathbf{Q}$. However, this projection has not always been fully included in theoretical calculations of the scattering pattern, which may help to account for the somewhat larger values of $J_{1}^{ \pm \pm}$proposed in Refs. [43,65].

Future magnetization studies at very low temperatures will be another important test for the presence of further-neighbor or anisotropic exchange interactions in $\mathrm{YbMgGaO}_{4}$. The presence of multiple phase transitions and magnetization plateaus in a moderate magnetic field [76] is expected in the case of a $X X Z$ triangular-lattice antiferromagnet $[77,78]$. This may also help to constrain the possible values of $J_{2}$ [79] and anisotropic exchanges, although exchange disorder may be very efficient at suppressing these features [80]. Because of their nonspin-conserving nature, dominant pseudodipolar interactions should manifest as an asymptotic approach to saturated magnetization, as recently observed in the candidate Kitaev spin liquid $\alpha-\mathrm{RuCl}_{3}$ [81]. Finally, we note that the role and extent of $\mathrm{CEF}$ and exchange disorder remains an outstanding issue in $\mathrm{YbMgGaO}_{4}$ with possible impact ranging from the mimicry of a spin liquid $[47,48,82,83]$ to disorder-induced entanglement [84-86].

\section{CONCLUSION}

In conclusion, combining time-domain terahertz spectroscopy and neutron scattering in the high-field regime of $\mathrm{YbMgGaO}_{4}$ yields the strongest constraints to date on possible mechanisms for the observed spin-liquid phenomenology. We note that a QSL regime is predicted for the spin-1/2 $J_{1}-J_{2}$ Heisenberg triangular-lattice antiferromagnet for $0.06 \lesssim J_{2} / J_{1} \lesssim 0.19$ [36-38]. While a previous analysis obtained $J_{2} / J_{1}=0.22$ [61], the present work yields a slightly smaller $J_{2} / J_{1}=0.18(7)$. Additionally, the ratio $J_{1}^{z z} / J_{1}^{ \pm}=1.75(5)$ suggests that $\mathrm{YbMgGaO}_{4}$ is more spin isotropic than previously thought $\left(J_{1}^{z z} / J_{1}^{ \pm}=1.16\right.$ [61]) with $J_{1}^{z z} / J_{1}^{ \pm}=2$ and 0 the Heisenberg and $X Y$ limits, respectively. We note, however, that subleading pseudodipolar interactions are also necessary to best explain our data. When combined with the likely presence of exchange disorder due to $\mathrm{Mg}^{2+}$ and $\mathrm{Ga}^{3+}$ disorder, this makes the spin-liquid mimicry $[47,48,82]$ or the $J_{1}-J_{2}$ quantum spin-liquid [36-38] mechanisms serious contenders to the various scenarios proposed to explain the physics of $\mathrm{YbMgGaO}_{4}$ thus far $[44,62,63]$.

\section{ACKNOWLEDGMENTS}

The authors would like to thank C. Batista, C. Broholm, H. Changlani, G. Chen, S. Chernyshev, S. Haravifard, J. Rau, and O. Starykh for helpful discussions. Research at Johns Hopkins University was funded by the U.S. DOE, Office of Basic Energy Sciences, Division of Materials Sciences and Engineering through Grant No. DE-FG0208ER46544. The work at Georgia Tech was supported by a Ralph E. Powe Junior Faculty Enhancement Award and by the National Science Foundation through Grant No. NSFDMR-1750186. The work at the University of Tennessee was supported by the National Science Foundation through Award No. NSF-DMR-1350002. The research at Oak Ridge National Laboratory's Spallation Neutron Source and High Flux Isotope Reactor was sponsored by the U.S. Department of Energy, Office of Basic Energy Sciences, Scientific User Facilities Division.

[1] P. A. Lee, An End to the Drought of Quantum Spin Liquids, Science 321, 1306 (2008).

[2] L. Balents, Spin Liquids in Frustrated Magnets, Nature (London) 464, 199 (2010).

[3] L. Savary and L. Balents, Quantum Spin Liquids: A Review, Rep. Prog. Phys. 80, 016502 (2017).

[4] Y. Zhou, K. Kanoda, and T.-K. Ng, Quantum Spin Liquid States, Rev. Mod. Phys. 89, 025003 (2017). 
[5] M. A. Levin and X.-G. Wen, String-Net Condensation: A Physical Mechanism for Topological Phases, Phys. Rev. B 71, 045110 (2005).

[6] T. Imai and Y.S. Lee, Do Quantum Spin Liquids Exist?, Phys. Today 69, No. 8, 30 (2016).

[7] T. Grover, Y. Zhang, and A. Vishwanath, Entanglement Entropy as a Portal to the Physics of Quantum Spin Liquids, New J. Phys. 15, 025002 (2013).

[8] Y. Shimizu, K. Miyagawa, K. Kanoda, M. Maesato, and G. Saito, Spin Liquid State in an Organic Mott Insulator with a Triangular Lattice, Phys. Rev. Lett. 91, 107001 (2003).

[9] F. L. Pratt, P. J. Baker, S. J. Blundell, T. Lancaster, S. OhiraKawamura, C. Baines, Y. Shimizu, K. Kanoda, I. Watanabe, and G. Saito, Magnetic and Non-Magnetic Phases of a Quantum Spin Liquid, Nature (London) 471, 612 (2011).

[10] K. Kanoda and R. Kato, Mott Physics in Organic Conductors with Triangular Lattices, Annu. Rev. Condens. Matter Phys. 2, 167 (2011).

[11] D. A. Tennant, R. A. Cowley, S. E. Nagler, and A. M. Tsvelik, Measurement of the Spin-Excitation Continuum in One-Dimensional $\mathrm{KCuF}_{3}$ Using Neutron Scattering, Phys. Rev. B 52, 13368 (1995).

[12] R. Coldea, D. A. Tennant, A. M. Tsvelik, and Z. Tylczynski, Experimental Realization of a 2 D Fractional Quantum Spin Liquid, Phys. Rev. Lett. 86, 1335 (2001).

[13] M. B. Stone, D. H. Reich, C. Broholm, K. Lefmann, C. Rischel, C. P. Landee, and M. M. Turnbull, Extended Quantum Critical Phase in a Magnetized Spin- $\frac{1}{2}$ Antiferromagnetic Chain, Phys. Rev. Lett. 91, 037205 (2003).

[14] R. Coldea, D. A. Tennant, E. M. Wheeler, E. Wawrzynska, D. Prabhakaran, M. Telling, K. Habicht, P. Smeibidl, and K. Kiefer, Quantum Criticality in an Ising Chain: Experimental Evidence for Emergent E8 Symmetry, Science 327, 177 (2010).

[15] M. Mourigal, M. Enderle, A. Klopperpieper, J.-S. Caux, A. Stunault, and H. M. Ronnow, Fractional Spinon Excitations in the Quantum Heisenberg Antiferromagnetic Chain, Nat. Phys. 9, 435 (2013).

[16] C. Balz, B. Lake, J. Reuther, H. Luetkens, R. Schonemann, T. Herrmannsdorfer, Y. Singh, A. T. M. Nazmul Islam, E. M. Wheeler, J. A. Rodriguez-Rivera, T. Guidi, G. G. Simeoni, C. Baines, and H. Ryll, Physical Realization of a Quantum Spin Liquid Based on a Complex Frustration Mechanism, Nat. Phys. 12, 942 (2016).

[17] A. Banerjee, J. Yan, J. Knolle, C. A. Bridges, M. B. Stone, M. D. Lumsden, D. G. Mandrus, D. A. Tennant, R. Moessner, and S.E. Nagler, Neutron Scattering in the Proximate Quantum Spin Liquid $\alpha-\mathrm{RuCl}_{3}$, Science 356, 1055 (2017).

[18] M. Oshikawa and I. Affleck, Electron Spin Eesonance in $S=1 / 2$ Antiferromagnetic Chains, Phys. Rev. B 65, 134410 (2002).

[19] K. Yu. Povarov, A. I. Smirnov, O. A. Starykh, S. V. Petrov, and A. Ya. Shapiro, Modes of Magnetic Resonance in the Spin-Liquid Phase of $\mathrm{Cs}_{2} \mathrm{CuCl}_{4}$, Phys. Rev. Lett. 107, 037204 (2011).

[20] V. N. Glazkov, M. Fayzullin, Yu. Krasnikova, G. Skoblin, D. Schmidiger, S. Mühlbauer, and A. Zheludev, ESR Study of the Spin Ladder with Uniform Dzyaloshinskii-Moriya Interaction, Phys. Rev. B 92, 184403 (2015).
[21] Z.-X. Luo, E. Lake, J.-W. Mei, and O. A. Starykh, Spinon Magnetic Resonance of Quantum Spin Liquids, Phys. Rev. Lett. 120, 037204 (2018).

[22] P. W. Anderson, Resonating Valence Bonds: A New Kind of Insulator?, Mater. Res. Bull. 8, 153 (1973).

[23] P. W. Anderson, The Resonating Valence Bond State in $\mathrm{La}_{2} \mathrm{CuO}_{4}$ and Superconductivity, Science 235, 1196 (1987).

[24] R. R. P. Singh and D. A. Huse, Three-Sublattice Order in Triangular and Kagome-Lattice Spin-Half Antiferromagnets, Phys. Rev. Lett. 68, 1766 (1992).

[25] P. Azaria, B. Delamotte, and D. Mouhanna, Spontaneous Symmetry Breaking in Quantum Frustrated Antiferromagnets, Phys. Rev. Lett. 70, 2483 (1993).

[26] L. Capriotti, A. E. Trumper, and S. Sorella, Long-Range Néel Order in the Triangular Heisenberg Model, Phys. Rev. Lett. 82, 3899 (1999).

[27] M. Poienar, F. Damay, C. Martin, J. Robert, and S. Petit, Spin Dynamics in the Geometrically Frustrated Multiferroic $\mathrm{CuCrO}_{2}$, Phys. Rev. B 81, 104411 (2010).

[28] S. E. Dutton, E. Climent-Pascual, P. W. Stephens, J. P. Hodges, A. Huq, C. L. Broholm, and R. J. Cava, Helical Magnetism and Structural Anomalies in Triangular Lattice $\alpha-\mathrm{SrCr}_{2} \mathrm{O}_{4}$, J. Phys. Condens. Matter 23, 246005 (2011).

[29] S. Toth, B. Lake, K. Hradil, T. Guidi, K. C. Rule, M. B. Stone, and A. T. M. N. Islam, Magnetic Soft Modes in the Distorted Triangular Antiferromagnet $\mathrm{CaCr}_{2} \mathrm{O}_{4}$, Phys. Rev. Lett. 109, 127203 (2012).

[30] J. Ma, Y. Kamiya, T. Hong, H. B. Cao, G. Ehlers, W. Tian, C. D. Batista, Z. L. Dun, H. D. Zhou, and M. Matsuda, Static and Dynamical Properties of the Spin-1/2 Equilateral Triangular-Lattice Antiferromagnet $\mathrm{Ba}_{3} \mathrm{CoSb}_{2} \mathrm{O}_{9}$, Phys. Rev. Lett. 116, 087201 (2016).

[31] S. Nakatsuji, Y. Nambu, H. Tonomura, O. Sakai, S. Jonas, C. Broholm, H. Tsunetsugu, Y. Qiu, and Y. Maeno, Spin Disorder on a Triangular Lattice, Science 309, 1697 (2005).

[32] J. P. Sheckelton, J. R. Neilson, D. G. Soltan, and T. M. McQueen, Possible Valence-Bond Condensation in the Frustrated Cluster Magnet $\mathrm{LiZn}_{2} \mathrm{Mo}_{3} \mathrm{O}_{8}$, Nat. Mater. 11, 493 (2012).

[33] B. Fåk, S. Bieri, E. Canévet, L. Messio, C. Payen, M. Viaud, C. Guillot-Deudon, C. Darie, J. Ollivier, and P. Mendels, Evidence for a Spinon Fermi Surface in the Triangular $S=1$ Quantum Spin Liquid $\mathrm{Ba}_{3} \mathrm{NiSb}_{2} \mathrm{O}_{9}$, Phys. Rev. B 95, 060402 (2017).

[34] Th. Jolicoeur, E. Dagotto, E. Gagliano, and S. Bacci, Ground-State Properties of the $S=1 / 2$ Heisenberg Antiferromagnet on a Triangular Lattice, Phys. Rev. B 42, 4800 (1990).

[35] L. O. Manuel and H. A. Ceccatto, Magnetic and Quantum Disordered Phases in Triangular-Lattice Heisenberg Antiferromagnets, Phys. Rev. B 60, 9489 (1999).

[36] P. H. Y. Li, R. F. Bishop, and C. E. Campbell, Quasiclassical Magnetic Order and Its Loss in a Spin-1/2 Heisenberg Antiferromagnet on a Triangular Lattice with Competing Bonds, Phys. Rev. B 91, 014426 (2015).

[37] Z. Zhu and S. R. White, Spin Liquid Phase of the $S=\frac{1}{2}$ $J_{1}-J_{2}$ Heisenberg Model on the Triangular Lattice, Phys. Rev. B 92, 041105 (2015). 
[38] Y. Iqbal, W.-J. Hu, R. Thomale, D. Poilblanc, and F. Becca, Spin Liquid Nature in the Heisenberg $J_{1}-J_{2}$ Triangular Antiferromagnet, Phys. Rev. B 93, 144411 (2016).

[39] G. Misguich, C. Lhuillier, B. Bernu, and C. Waldtmann, Spin-Liquid Phase of the Multiple-Spin Exchange Hamiltonian on the Triangular Lattice, Phys. Rev. B 60, 1064 (1999).

[40] R. V. Mishmash, J. R. Garrison, S. Bieri, and C. Xu, Theory of a Competitive Spin Liquid State for Weak Mott Insulators on the Triangular Lattice, Phys. Rev. Lett. 111, 157203 (2013).

[41] Y.-D. Li, X. Wang, and G. Chen, Anisotropic Spin Model of Strong Spin-Orbit-Coupled Triangular Antiferromagnets, Phys. Rev. B 94, 035107 (2016).

[42] Y.-D. Li, Y.-M. Lu, and G. Chen, The Spinon Fermi Surface U(1) Spin Liquid in a Spin-Orbit-Coupled Triangular Lattice Mott Insulator $\mathrm{YbMgGaO}_{4}$, Phys. Rev. B 96, 054445 (2017).

[43] Y.-D. Li, Y. Shen, Y. Li, J. Zhao, and G. Chen, The Effect of Spin-Orbit Coupling on the Effective-Spin Correlation in $\mathrm{YbMgGaO}_{4}$, Phys. Rev. B 97, 125105 (2018).

[44] Y.-D. Li and G. Chen, Detecting Spin Fractionalization in a Spinon Fermi Surface Spin Liquid, Phys. Rev. B 96, 075105 (2017).

[45] C. Liu, R. Yu, and X. Wang, Semiclassical Ground-State Phase Diagram and Multi-Q Phase of a Spin-OrbitCoupled Model on Triangular Lattice, Phys. Rev. B 94, 174424 (2016).

[46] Q. Luo, S. Hu, B. Xi, J. Zhao, and X. Wang, Ground-State Phase Diagram of an Anisotropic Spin- $\frac{1}{2}$ Model on the Triangular Lattice, Phys. Rev. B 95, 165110 (2017).

[47] Z. Zhu, P. A. Maksimov, S. R. White, and A. L. Chernyshev, Disorder-Induced Mimicry of a Spin Liquid in $\mathrm{YbMgGaO}_{4}$, Phys. Rev. Lett. 119, 157201 (2017).

[48] Z. Zhu, P. A. Maksimov, S. R. White, and A. L. Chernyshev, Topography of Spin Liquids on a Triangular Lattice, Phys. Rev. Lett. 120, 207203 (2018).

[49] K. A. Ross, L. Savary, B. D. Gaulin, and L. Balents, Quantum Excitations in Quantum Spin Ice, Phys. Rev. X 1, 021002 (2011).

[50] S. Onoda and Y. Tanaka, Quantum Fluctuations in the Effective Pseudospin- $\frac{1}{2}$ Model for Magnetic Pyrochlore Oxides, Phys. Rev. B 83, 094411 (2011).

[51] L. Pan, S. K. Kim, A. Ghosh, C. M. Morris, K. A. Ross, E. Kermarrec, B. D. Gaulin, S. M. Koohpayeh, O. Tchernyshyov, and N. P. Armitage, Low-Energy Electrodynamics of Novel Spin Excitations in the Quantum Spin Ice $\mathrm{Yb}_{2} \mathrm{Ti}_{2} \mathrm{O}_{7}$, Nat. Commun. 5, 4970 (2014).

[52] L. Pan, N. J. Laurita, K. A. Ross, B. D. Gaulin, and N. P. Armitage, A Measure of Monopole Inertia in the Quantum Spin Ice $\mathrm{Yb}_{2} \mathrm{Ti}_{2} \mathrm{O}_{7}$, Nat. Phys. 12, 361 (2016).

[53] J. D. Thompson, P. A. McClarty, D. Prabhakaran, I. Cabrera, T. Guidi, and R. Coldea, Quasiparticle Breakdown and Spin Hamiltonian of the Frustrated Quantum Pyrochlore $\mathrm{Yb}_{2} \mathrm{Ti}_{2} \mathrm{O}_{7}$ in a Magnetic Field, Phys. Rev. Lett. 119, 057203 (2017).

[54] Y. Li, H. Liao, Z. Zhang, S. Li, F. Jin, L. Ling, L. Zhang, Y. Zou, L. Pi, Z. Yang, J. Wang, Z. Wu, and Q. Zhang, Gapless Quantum Spin Liquid Ground State in the Two-Dimensional
Spin-1/2 Triangular Antiferromagnet $\mathrm{YbMgGaO}_{4}$, Sci. Rep. 5, 16419 (2015).

[55] Y. Li, G. Chen, W. Tong, L. Pi, J. Liu, Z. Yang, X. Wang, and Q. Zhang, Rare-Earth Triangular Lattice Spin Liquid: A Single-Crystal Study of $\mathrm{YbMgGaO}_{4}$, Phys. Rev. Lett. 115, 167203 (2015).

[56] T.-H. Han, J. S. Helton, S. Chu, D. G. Nocera, J. A. Rodriguez-Rivera, C. Broholm, and Y. S. Lee, Fractionalized Excitations in the Spin-Liquid State of a KagomeLattice Antiferromagnet, Nature (London) 492, 406 (2012).

[57] A. Zorko, S. Nellutla, J. van Tol, L. C. Brunel, F. Bert, F. Duc, J.-C. Trombe, M. A. de Vries, A. Harrison, and P. Mendels, Dzyaloshinsky-Moriya Anisotropy in the Spin-1/2 Kagome Compound $\mathrm{ZnCu}_{3}(\mathrm{OH})_{6} \mathrm{Cl}_{2}$, Phys. Rev. Lett. 101, 026405 (2008).

[58] M. R. Norman, Colloquium: Herbertsmithite and the Search for the Quantum Spin Liquid, Rev. Mod. Phys. 88, 041002 (2016).

[59] Y. Li, D. Adroja, P. K. Biswas, P. J. Baker, Q. Zhang, J. Liu, A. A. Tsirlin, P. Gegenwart, and Q. Zhang, Muon Spin Relaxation Evidence for the U(1) Quantum Spin-Liquid Ground State in the Triangular Antiferromagnet $\mathrm{YbMgGaO}_{4}$, Phys. Rev. Lett. 117, 097201 (2016).

[60] Y. Xu, J. Zhang, Y. S. Li, Y. J. Yu, X. C. Hong, Q. M. Zhang, and S. Y. Li, Absence of Magnetic Thermal Conductivity in the Quantum Spin-Liquid Candidate $\mathrm{YbMgGaO}_{4}$, Phys. Rev. Lett. 117, 267202 (2016).

[61] J. A. M. Paddison, M. Daum, Z. Dun, G. Ehlers, Y. Liu, M. B. Stone, H. Zhou, and M. Mourigal, Continuous Excitations of the Triangular-Lattice Quantum Spin Liquid $\mathrm{YbMgGaO}_{4}$, Nat. Phys. 13, 117 (2017).

[62] Y. Shen, Y.-D. Li, H. Wo, Y. Li, S. Shen, B. Pan, Q. Wang, H. C. Walker, P. Steffens, M. Boehm, Y. Hao, D. L. Quintero-Castro, L. W. Harriger, M. D. Frontzek, L. Hao, S. Meng, Q. Zhang, G. Chen, and J. Zhao, Evidence for a Spinon Fermi Surface in a Triangular-Lattice Quantum Spin Liquid Candidate, Nature (London) 540, 559 (2016).

[63] Y. Li, D. Adroja, D. Voneshen, R. I. Bewley, Q. Zhang, A. A. Tsirlin, and P. Gegenwart, Nearest-Neighbour Resonating Valence Bonds in $\mathrm{YbMgGaO}_{4}$, Nat. Commun. 8, 15814 (2017).

[64] Y. Li, D. Adroja, R. I. Bewley, D. Voneshen, A. A. Tsirlin, P. Gegenwart, and Q. Zhang, Crystalline Electric-Field Randomness in the Triangular Lattice Spin-Liquid $\mathrm{YbMgGaO}_{4}$, Phys. Rev. Lett. 118, 107202 (2017).

[65] S. Tóth, K. Rolfs, A. R. Wildes, and C. Rüegg, Strong Exchange Anisotropy in $\mathrm{YbMgGaO}_{4}$ from Polarized Neutron Diffraction, arXiv:1705.05699.

[66] J. G. Rau and M. J. P. Gingras, Frustration and Anisotropic Exchange in Ytterbium Magnets with Edge-Shared Octahedra, arXiv:1802.03024.

[67] C. M. Morris, R. Valdés Aguilar, A. Ghosh, S. M. Koohpayeh, J. Krizan, R. J. Cava, O. Tchernyshyov, T. M. McQueen, and N. P. Armitage, Hierarchy of Bound States in the One-Dimensional Ferromagnetic Ising Chain $\mathrm{CoNb}_{2} \mathrm{O}_{6}$ Investigated by High-Resolution Time-Domain Terahertz Spectroscopy, Phys. Rev. Lett. 112, 137403 (2014).

[68] See Supplemental Material at http://link.aps.org/ supplemental/10.1103/PhysRevX.8.031001 for further details about the sample preparation, experimental setup, 
analysis of the INS, THz and FTIR data and expressions for the spin-wave dispersion and linewidth calculations.

[69] G. Ehlers, A. A. Podlesnyak, J. L. Niedziela, E. B. Iverson, and P. E. Sokol, The New Cold Neutron Chopper Spectrometer at the Spallation Neutron Source: Design and Performance, Rev. Sci. Instrum. 82, 085108 (2011).

[70] N. P. Armitage, Constraints on Jones Transmission Matrices from Time-Reversal Invariance and Discrete Spatial Symmetries, Phys. Rev. B 90, 035135 (2014).

[71] N. J. Laurita, J. Deisenhofer, L. D. Pan, C. M. Morris, M. Schmidt, M. Johnsson, V. Tsurkan, A. Loidl, and N. P. Armitage, Singlet-Triplet Excitations and Long-Range Entanglement in the Spin-Orbital Liquid Candidate $\mathrm{FeSc}_{2} \mathrm{~S}_{4}$, Phys. Rev. Lett. 114, 207201 (2015).

[72] D. L. Huber, Effect of the Pseudodipolar Interaction on Critical Spin Dynamics in Cubic Ferromagnets, Solid State Commun. 48, 831 (1983).

[73] J. H. van Vleck, On the Anisotropy of Cubic Ferromagnetic Crystals, Phys. Rev. 52, 1178 (1937).

[74] R. Kubo and K. Tomita, A General Theory of Magnetic Resonance Absorption, J. Phys. Soc. Jpn. 9, 888 (1954).

[75] T. G. Castner and M. S. Seehra, Antisymmetric Exchange and Exchange-Narrowed Electron-Paramagnetic-Resonance Linewidths, Phys. Rev. B 4, 38 (1971).

[76] O. A Starykh, Unusual Ordered Phases of Highly Frustrated Magnets: A Review, Rep. Prog. Phys. 78, 052502 (2015).

[77] T. Susuki, N. Kurita, T. Tanaka, H. Nojiri, A. Matsuo, K. Kindo, and H. Tanaka, Magnetization Process and Collective Excitations in the $S=1 / 2$ Triangular-Lattice Heisenberg Antiferromagnet $\mathrm{Ba}_{3} \mathrm{CoSb}_{2} \mathrm{O}_{9}$, Phys. Rev. Lett. 110, 267201 (2013).

[78] Y. Kamiya, L. Ge, T. Hong, Y. Qiu, D. L. Quintero-Castro, H. B. Cao, M. Matsuda, C. D. Batista, M. Mourigal, H. D. Zhou, and J. Ma, The Nature of Spin Excitations in the
One-Third Magnetization Plateau Phase of $\mathrm{Ba}_{3} \mathrm{CoSb}_{2} \mathrm{O}_{9}$, arXiv:1701.07971.

[79] M. Ye and A. V. Chubukov, Quantum Phase Transitions in the Heisenberg $J_{1}-J_{2}$ Triangular Antiferromagnet in a Magnetic Field, Phys. Rev. B 95, 014425 (2017).

[80] A. I. Smirnov, T. A. Soldatov, O. A. Petrenko, A. Takata, T. Kida, M. Hagiwara, A. Ya. Shapiro, and M. E. Zhitomirsky, Order by Quenched Disorder in the Model Triangular Antiferromagnet $\mathrm{RbFe}\left(\mathrm{MoO}_{4}\right)_{2}$, Phys. Rev. Lett. 119, 047204 (2017).

[81] S. Winter, K. Riedl, D. Kaib, R. Coldea, and R. Valenti, Probing $\alpha-\mathrm{RuCl}_{3}$ Beyond Magnetic Order: Effects of Temperature and Magnetic Field, Phys. Rev. Lett. 120, 077203 (2018).

[82] E. Parker and L. Balents, Finite-Temperature Behavior of a Classical Spin-Orbit-Coupled Model for $\mathrm{YbMgGaO}_{4}$ with and without Bond Disorder, Phys. Rev. B 97, 184413 (2018).

[83] Z. Ma et al., Spin-Glass Ground State in a TriangularLattice Compound $\mathrm{YbZnGaO}_{4}$, Phys. Rev. Lett. 120, 087201 (2018).

[84] L. Savary and L. Balents, Disorder-Induced Quantum Spin Liquid in Spin Ice Pyrochlores, Phys. Rev. Lett. 118, 087203 (2017).

[85] J.-J. Wen, S. M. Koohpayeh, K. A. Ross, B. A. Trump, T. M. McQueen, K. Kimura, S. Nakatsuji, Y. Qiu, D. M. Pajerowski, J. R. D. Copley, and C. L. Broholm, Disordered Route to the Coulomb Quantum Spin Liquid: Random Transverse Fields on Spin Ice in $\mathrm{Pr}_{2} \mathrm{Zr}_{2} \mathrm{O}_{7}$, Phys. Rev. Lett. 118, 107206 (2017).

[86] I. Kimchi, A. Nahum, and T. Senthil, Valence Bonds in Random Quantum Magnets: Theory and Application to $\mathrm{YbMgGaO}_{4}$, arXiv:1710.06860.

Correction: One reference (Ref. [83]) and its citations in text were missing and have been inserted. 